\title{
Survey on Machine Learning for Crop Prediction Using Soil Nutrients Data
}

\section{Author: R. Kesavamoorthy'; ${ }^{1}$ Sameer Paudel ${ }^{2}$; Riyaz Nakarmi ${ }^{3}$; Prashant Giri ${ }^{4}$; Shantanu Bahadur Karki ${ }^{5}$}

\author{
Affiliation: Jain University ${ }^{1}$; Jain University ${ }^{2}$; Jain University ${ }^{3}$; Jain University ${ }^{4}$; Jain University ${ }^{5}$ \\ E-mail: r.kesavamoorthy@jainuniversity.ac.in ${ }^{1}$; 17btrcs119@jainuniversity.ac.in ${ }^{2}$; \\ 17btrcs117@jainuniversity.ac.in ${ }^{3} ; 17 b t r c s 115 @ j a i n u n i v e r s i t y . a c . i n{ }^{4}$; \\ 17btrcs121@jainuniversity.ac.in ${ }^{5}$
}

DOI: 10.26821/IJSHRE.9.1.2021.9101

\begin{abstract}
Agriculture is the primary source of livelihood which forms the backbone of many different countries. Current challenges in agriculture field due to traditional farming system necessitate farmers to be equipped with modern farming system. Machine learning technique helps to predict crop yield in agriculture that can help framers. In this system, we are using machine learning techniques which help to suggest the crops according to soil data. The model predicts and suggest most accurate crop based on the soil attributes. This prediction will help farmer to predict the yield of the crop before cultivating and thus help them to make appropriate decisions. Machine learning technique provides efficient framework for data driven decision making. This model provides set machine learning techniques to help farmers grow crops depending on their field important attributes.
\end{abstract}

Keywords: agriculture, crop, soil, nutrient, machine learning, prediction

\section{INTRODUCTION}

Agriculture is the main source of livelihoods of the majority of the people in South Asia. Agriculture is the backbone of the economy in a country like India where more than half of the population is involved in agriculture related work and plays an important role in the development of the country. India being one of the most populated countries has increasing demand for food so adopting new technologies in the field of agriculture is a must. But farmers in India still lack knowledge about soil, crops and new technologies due to which there is decline in production and satisfactory results are not achieved. Agriculture sector in India is facing a problem to maximize the production of crops by using traditional methods. So advancement and use of new technology in the agricultural field is essential and helps farmers to increase their profit.

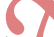

As we know soil is the most essential thing in agriculture. The quality and quantity of the crops are directly proportional the quality of the soil. So we need to test the soil and find out what types of crop can be planted in the given area. The quality of the soil can be analyzed using the nutrients present in the soil such as Nitrogen (N), Potassium (k), Ca (Calcium), and Phosphorus (P). The soil data can be analyzed by the use of machine learning technique. The system is created using machine learning algorithm and it predicts appropriate crops to the farmers depending on the quality of the soil in the area. Machine learning concentrates on the creation of computer programs that can access data and use it to learn from that. Machine learning allows building models from sample data and gives the ability to take decision automatically according to past experiences.

We are motivated in building a system that helps farmers and all those involved in the agricultural field to decide on what crops to be ploughed in what area. This dataset consists of the available nutrient for farmers' soil for a particular region. The soil type is then decided by the nutrient data for plantation. The soil type then helps farmers decide on which crops to plant in particular areas for better harvest which in turn increases yield of crops and also correspondingly increases the earnings of farmers respectively. We use

R. Kesavamoorthy; Sameer Paudel; Riyaz Nakarmi; Prashant Giri; Shantanu Bahadur Karki, Volume 9 Issue

1, pp 1-4, January 2021 
Volume 9 Issue 1 January 2021

machine learning to create the system which concentrates on the creation of computer programs that can access data and use it to learn from that. Machine learning allows different building models from given sample data and give the ability to take decision automatically according to past experiences.

\section{RELATED WORKS}

This section will focus on the various works related to the prediction of soil quality using techniques related to Machine Learning.

\subsection{Prediction of Soil Quality Using Machine Leaning Techniques}

\subsubsection{Proposed Technique \& Features:}

Suggests a software enabled solution considering crucial soil parameters and soil factors to predict the soil quality. This project predicts the quality of soil by using machine learning algorithms like Decision Tree Algorithm and Random Forest Algorithm.

\subsubsection{Advantages:}

Fast processing and predictions in real time. Large and complicated process environments provide constant quality. Automation of tasks is easily implemented.

\subsubsection{Limitations:}

This research will be biased towards modeling the qualities of the particular testers who generated the data, which may differ from the ratings given by different testers.

\subsection{Crop Monitoring and Recommendation System using Machine Learning and IOT}

\subsubsection{Proposed Technique \& Features:}

Implementation of a smart agricultural system that uses advantages of cutting edge technologies such as IoT, Sensor network and data analysis to help farmers enhance the way farming. This system uses Crop Monitoring System which contains six components namely Sensors, NodeMcu, ThingSpeak Cloud, Android Application, ML Model, and Crop Assessment and Suggestions.

\subsubsection{Advantages:}

The proposed system provides a very useful Android based application that facilitates an interface for farmers to monitor soil conditions and generate recommendations to improve crop production.

\subsubsection{Limitations:}

The prediction might not work during natural calamities since it doesn't take them into consideration.

\subsection{Crop Prediction based on Soil Classification using Machine Learning with Classifier Ensemble \\ 2.3.1 Proposed Technique \& Features:}

Using two datasets, the first being a soil dataset an the second being crop dataset. Soil dataset has attributes like $\mathrm{pH}$, nitrogen, phosphorous, potassium, soil type and the crop dataset has attributes like soil type and crop list. Five algorithms used in this are Support Vector Machine, Bagged Tree, Adaboost, Naive Bayes, and Artificial Neural Network. Ensemble method to increase accuracy.

\subsubsection{Advantages:}

Five different algorithms are used in system that gives us best prediction. Usage of ensemble method, this model generates accurate model than base model.

\subsubsection{Limitations:}

This method used in this system is time consuming (ensemble method) changes in the soil composition due to natural phenomenon can disrupt data collection.

\subsection{Crop prediction using Machine Learning 2.4.1 Proposed Technique \& Features:}

Solve agriculture problem by monitoring agriculture area on basis of soil properties.

Different algorithms models are used. Simple and easy to understand.

\subsubsection{Advantages:}

Large dataset. Different algorithms are used which have different accuracies. This speed up the process.

\subsubsection{Limitations:}

Disaster like flood and soil erosion changes the soil composition so prediction may not work.

\subsection{Soil classification and crop suggestion using machine learning algorithm 2.5.1 Proposed Technique \& Features:}

Suggest the most suitable for a specific land based on the analysis of the data on certain affecting parameters like temperature, humidity, air quality and $\mathrm{PH}$ of soil 
Volume 9 Issue 1 January 2021

using machine learning. This project uses parameter like temperature, humidity, air quality, and $\mathrm{pH}$ of soil to suggest crop.

\subsubsection{Advantages:}

The UI of the project is designed such that it is easily understandable by the common people.

\subsubsection{Limitations:}

Since many parameters are used so accuracy of prediction is less.

\subsection{Demand Based Crop Recommendation System for Farmers \\ 2.6.1 Proposed Technique \& Features:}

Making use of a sliding window non-linear regression technique to predict based on different factors affecting agricultural production such as rainfall, temperature, market prices, area of land and past yield of a crop. The system uses soil data and the demand of crops in the regional area to predict suitable crops to the farmers.

\subsubsection{Advantages:}

The system had in-built translator so people who might not be able to understand English can also use it to the fullest.

\subsubsection{Limitations:}

The system isn't $100 \%$ accurate so there is a chance of some miscalculation which might lead to the production of crops not being to the required level.

\subsection{Crop prediction system using machine learning algorithms \\ 2.7.1 Proposed Technique \& Features:}

The project work introduces an efficient recommendation system using classifier models to recommend the crops. This system uses combination pf classifier models like Decision tree and Naive Bayes to improve efficiency of the prediction. From the yield graphs the best time of sowing, plant growth and harvesting of plant can also be found out along with prediction for crops. The system not only predicts crop prediction based on soil parameters but also takes rainfall into account.

\subsubsection{Advantages:}

The combination of classification algorithm are better performing than use of single classifier model. The system is scalable as it can be used to test on different crops.

\subsubsection{Limitations:}

Different factors make the prediction difficult and more time consuming.

\subsection{Crop Recommendation System to Maximize Crop Yield using Machine Learning Technique \\ 2.8.1 Proposed Technique \& Features:}

This project model recommends the system to maximize the crop using different parameter like $\mathrm{pH}$, soil color, permeability, Drainage, Water holding. This model uses Ensemble technique for prediction. Learners used in this system are support vector, Naïve Bayes, Random forest, Multi-layer perception. This system basically uses all soil parameters.

\subsubsection{Advantages:}

As this system uses the ensemble method this gives us accurate result. Variety of learners are used in this model which help us to maximize the crop yield.

\subsubsection{Limitations:}

Natural disaster like soil erosion and flood makes the prediction difficult as this system fully depends on soils parameter.

\section{CONCLUSION AND FUTURE SCOPE}

We studied several research papers available online related to our project. In these proposed research works the Classification of Soils and prediction of yield has been done on the basis of Soil Composition parameters which is one of subsets for crop Yield Prediction. All these papers are based on crop yield prediction on the basis of soil parameters but they use different algorithms, have different features and different accuracies. We have incorporated the study from these papers to initiate and continue work in our system.

\section{ACKNOWLEDGEMENT}

It is a great pleasure for us to acknowledge the assistance and support of faculty members at JAIN (Deemed to be) University. Likewise our supportive parents who have been supporting for the successful completion of this project work. 
Volume 9 Issue 1 January 2021

\section{REFERENCES}

[1]. Rao, T Venkat Narayana, and Gaddam Rishitha Reddy. "Prediction Of Soil Quality Using Machine Leaning Techniques." International Journal of Scientific \& Technology Research 08.11 (2019).

[2]. Reddy, R. Pallavi, B. Vinitha, K. Rishita, and K. Pranavi. "Crop Monitoring and Recommendation System Using Machine Learning and IOT." International Journal of Innovative Technology and Exploring Engineering (IJITEE) 9.9 (2020).

[3]. Waikar, Vrushali C., Sheetal Y. Thorat, Ashlesha A. Ghunte, Priya P. Rajput, and Mahesh S. Shinde. "Crop Prediction Based on Soil Classification Using Machine Learning with Classifier Ensembling." International Research Journal of Engineering and Technology (IRJET) 07.05 (2020).

[4]. Thomas, Kevin Thomas Tom, Varsha S, Merin Mary Saji, Lisha Varghese, and Er. Jinu Thomas. " Crop Prediction Using Machine Learning." International Journal of Future Generation Communication and Networking (2020).

[5]. Mule, Snehal, and Mandar Sohani, Prof. "Soil Classification and Crop Suggestion Using
Machine Learning Algorithm." Journal of Emerging Technologies and Innovative Research (JETIR) 7.4 (2020).

[6]. Raja, S.Kanaga Subh, R. Rishi, E. Sundaresan, and V. Srijit. "Demand Based Crop Recommender System for Farmers." IEEE Technological Innovations in ICT for Agriculture and Rural Development (2017).

[7]. Patil, Pavan, Virendra Panpatil, and Prof. Shrikant Kokate. "Crop Prediction System Using Machine Learning Algorithms." International Research Journal of Engineering and Technology (2020).

[8]. Rajak, Rohit Kumar, Ankit Pawar, Mitalee Pendke, Suresh Rathod, Pooja Shinde, and Avinash Devare. "Crop Recommendation System to Maximize Crop Yield Using Machine Learning Technique." International Research Journal of Engineering and Technology (2017)

[9]. Jajur, S. Mamatha, Soumya N. G., and G. T. Raju. "Crop Recommendation Using Machine Learning Techniques." International Journal of Innovative Technology and Exploring Engineering (2019). 